\title{
Production of silica aerogel radiator tiles for the HELIX RICH detector
}

P. Allison ${ }^{1}$, J. J. Beatty ${ }^{1}$, L. Beaufore ${ }^{2}$, Y. Chen ${ }^{3}$, S. Coutu ${ }^{3}$, E. Ellingwood ${ }^{4}$, M. Gebhard ${ }^{5}$, N. Green ${ }^{6}$, D. Hanna ${ }^{4}$, B. Kunkler ${ }^{5}$, I. Mognet $^{3}$, R. Mbarek ${ }^{2}$, K. McBride ${ }^{1}$, K. Michaels ${ }^{5}$, D. Müller ${ }^{2}$, J. Musser ${ }^{5}$, S. Nutter ${ }^{7}$, S. O’Brien ${ }^{4}$, N. Park ${ }^{8}$,

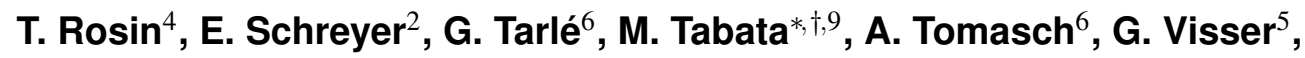
S. P. Wakely ${ }^{2}$, T. Werner ${ }^{2}$, I. Wisher ${ }^{2}$, and M. Yu ${ }^{3}$

${ }^{1}$ The Ohio State University, Columbus, OH 43210 USA

${ }^{2}$ University of Chicago, Chicago, IL 60637 USA

${ }^{3}$ Pennsylvania State University, University Park, PA 16802 USA

${ }^{4}$ Mc Gill University, Montreal, QC H3A 2T8, Canada

${ }^{5}$ Indiana University, Bloomington, IN 47405 USA

${ }^{6}$ University of Michigan, Ann Arbor, MI 48109 USA

${ }^{7}$ Northern Kentucky University, Highland Heights, KY 41099 USA

${ }^{8}$ University of Wisconsin-Madison, Madison, WI 53706 USA

${ }^{9}$ Chiba University, Chiba, 263-8522, Japan

\begin{abstract}
A hydrophobic, highly transparent silica aerogel with a refractive index of $\sim 1.15$ was developed using sol-gel polymerization, pin drying, and supercritical carbon dioxide solvent extraction technologies. A total of 96 monolithic tiles with dimensions of $11 \mathrm{~cm} \times 11 \mathrm{~cm} \times 1 \mathrm{~cm}$ were mass produced with a high crack-free yield for use as Cherenkov radiators to be installed in the proximityfocusing ring-imaging Cherenkov (RICH) detector. The RICH detector, containing 36 aerogel tiles, will be installed in the High Energy Light Isotope eXperiment (HELIX) spectrometer and used to measure the velocity of cosmic-ray particles. HELIX is a balloon-borne experimental program designed to measure the chemical and isotopic abundances of light cosmic-ray nuclei. A water-jet cut test of the aerogel tiles and a gluing test of the trimmed tiles with dimensions of 10 $\mathrm{cm} \times 10 \mathrm{~cm} \times 1 \mathrm{~cm}$ in an aluminum frame were successful in the context of integration into the radiator module.
\end{abstract}

36th International Cosmic Ray Conference -ICRC2019-

July 24th - August 1st, 2019

Madison, WI, U.S.A.

\footnotetext{
${ }^{*}$ Speaker.

†E-mail: makoto@hepburn.s.chiba-u.ac.jp
} 


\section{Introduction}

HELIX [U, [ [] is a balloon-borne experiment designed to measure the chemical and isotopic abundances of light cosmic-ray nuclei, particularly the ${ }^{10} \mathrm{Be} /{ }^{9} \mathrm{Be}$ ratio over the energy range from $0.2 \mathrm{GeV} / \mathrm{n}$ to more than $3 \mathrm{GeV} / \mathrm{n}$, which is a key measurement for constraining cosmic-ray propagation models. The HELIX detector (with a flight scheduled during NASA's 2020/21 Antarctic balloon campaign) is a mass spectrometer based on particle momentum and velocity measurements. The detector system includes a 1-T superconducting magnet, a high-resolution tracking system, time-of-flight counters, and a RICH detector. The HELIX RICH system is an aerogelbased proximity-focusing detector with an expansion length of $50 \mathrm{~cm}$ [B]]. Hydrophobic silica aerogel and silicon photomultipliers were chosen as the Cherenkov radiators and photosensors, respectively. Highly transparent aerogel tiles with an ultrahigh refractive index of $\sim 1.15$ and dimensions of $10 \mathrm{~cm} \times 10 \mathrm{~cm} \times 1 \mathrm{~cm}$ were custom-made using recently developed pin-drying production technology [䧃]. In this paper, we report results from the full-scale mass production of approximately 100 aerogel tiles (36 of them to be used as flight tiles) and their initial optical assessment.

\section{Production method}

The refractive index (i.e., density) of silica aerogels, which is determined by the volume ratio of cross-linked silica particles and pores, can be controlled via a wet-gel synthesis process based on a sol-gel method. In the synthesis step, the primary reactants (i.e., the silica precursor and water), diluent solvents, and catalysts were mixed according to a recipe given by a quasi-empirical formula to obtain a target index. This recipe is based on the fact that the volume shrinkage of a wet gel in the production process can be minimized by aging it for a sufficient duration at a certain temperature and applying a supercritical fluid-extraction/drying method. In this conventional procedure, it is possible to produce aerogel tiles with indices of up to $\sim 1.14$; however, it is difficult to attain sufficiently transparent tiles with indices greater than $\sim 1.10$ [[]].
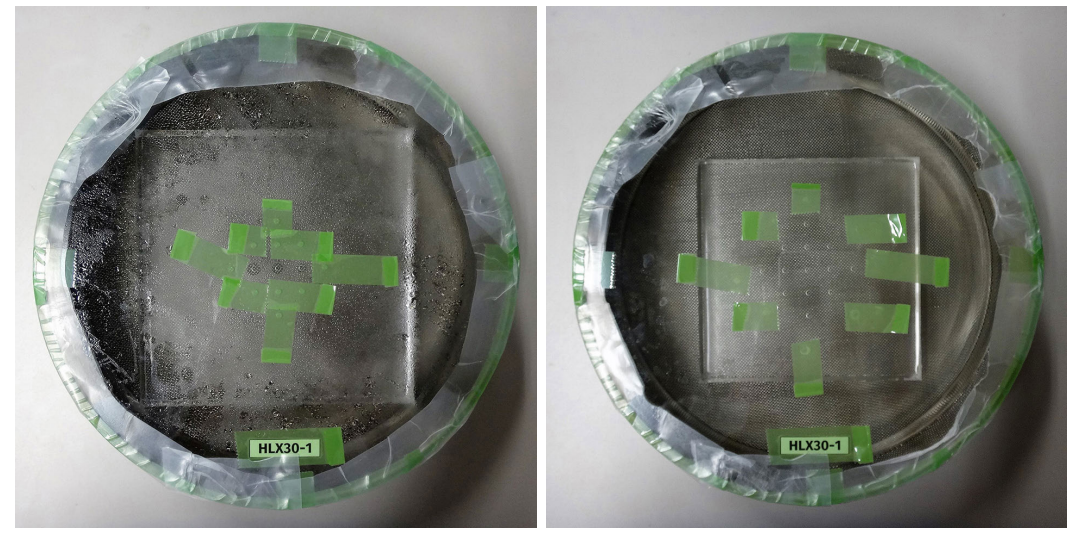

Figure 1: At the beginning (left) and end (right) of the pin-drying process. The pin container comprised a stainless-steel sieve for the body, glass top plate, and aluminum bottom plate. The wet-gel tile was placed on the mesh of the sieve. Each plate had 13 2-mm diameter pinholes. To open and close the pinholes, chemical-resistant tapes (green) were used. 
In the present study, hydrophobic, highly transparent aerogel tiles with a nominal index of 1.15 were produced using a recently developed pinhole drying technology, which is also called a pin-drying method. The aging process was shortened and replaced by a pin-drying step to densify the wet gel prior to the extraction of the supercritical solvent. The densification of the wet gel via shrinkage (without cracking owing to very slow, partial solvent evaporation) was achieved by enclosing the gel in a semi-sealed container with pinholes (called a pin container) and placing the container in a temperature-controlled environment (see Figure $\mathrm{W}$ ). To achieve uniform shrinkage, the weight reduction rate of the wet gel was indirectly monitored while varying the number of open pinholes. The pin drying was continued until the gel was reduced to a predetermined weight to obtain the target index. The retrieved wet gel was then subjected to a hydrophobic treatment. Finally, the wet gel was rendered to an aerogel via the supercritical carbon dioxide extraction method. In the present case, the initial wet gel targeting an aerogel with an index of 1.11 (in the case of the usual solvent extraction via the supercritical phase) was synthesized and pin dried for 2-2.5 months to obtain an index of $\sim 1.15$ (after the supercritical extraction).

\section{Mass-production results}

The final 96-tile mass production began in March 2018 and was completed in December of the same year. Once per day, four wet-gel tiles with dimensions of $135 \mathrm{~mm} \times 135 \mathrm{~mm} \times 12$ $\mathrm{mm}$ were synthesized in a plastic mold using an organic diluent solvent and were individually enclosed in a pin container by detaching the mold after $1 \mathrm{~h}$ of aging. After the pin drying, the wet gel was soaked in ethanol and subjected to a hydrophobic treatment using silazane. The 96 wet-gel tiles were divided into six batches (i.e., 16 tiles per batch), and 16 tiles were placed in an autoclave, which is a part of an in-house-constructed supercritical fluid-extraction system. The ethanol solvent was exchanged for liquefied carbon dioxide in the sealed autoclave, and the wet-gel tiles were completely dried via the supercritical phase of carbon dioxide while maintaining their volume.

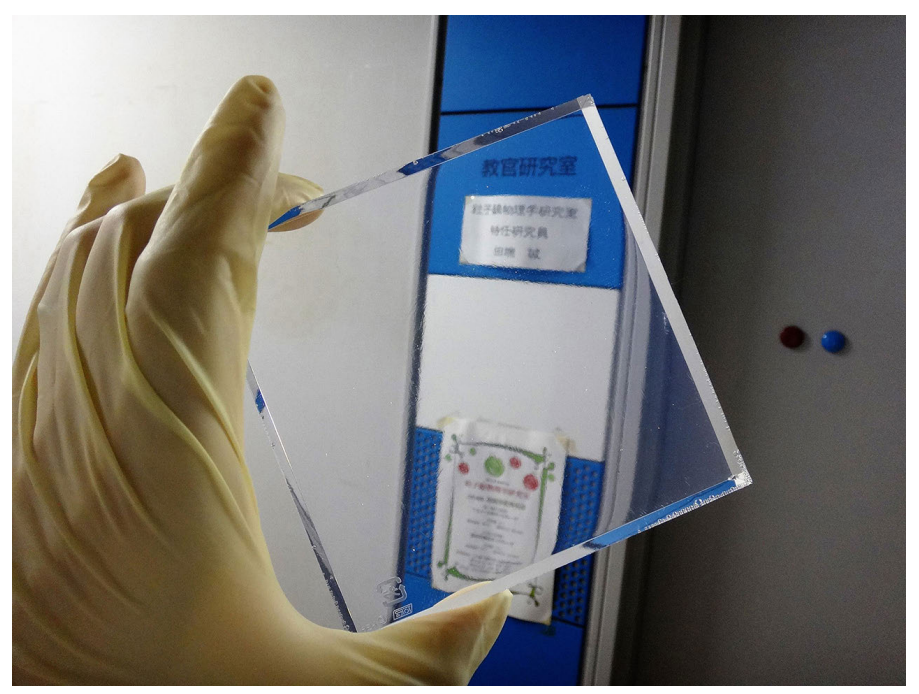

Figure 2: The first aerogel tile as prepared via mass production from Ref. [目]). 


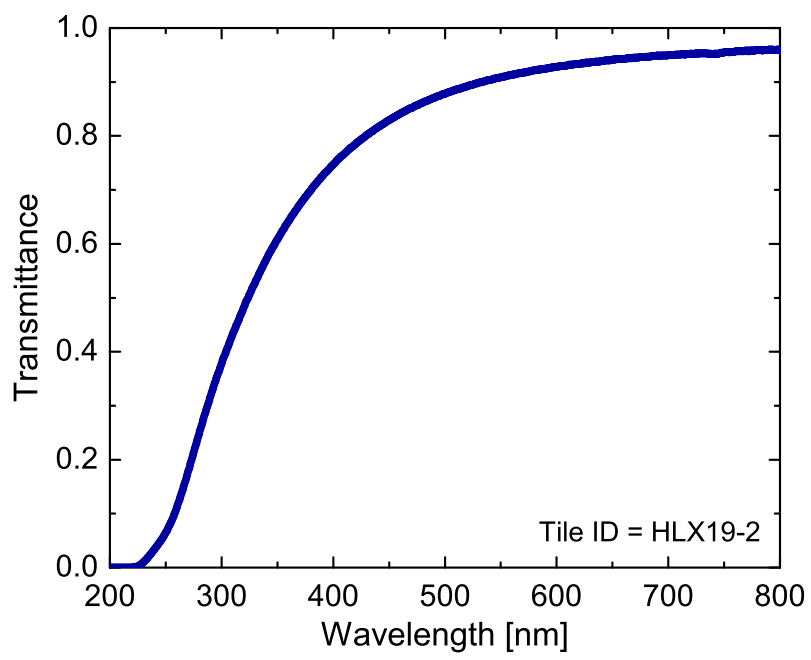

Figure 3: UV-vis transmittance spectrum of the first mass-produced aerogel tile.

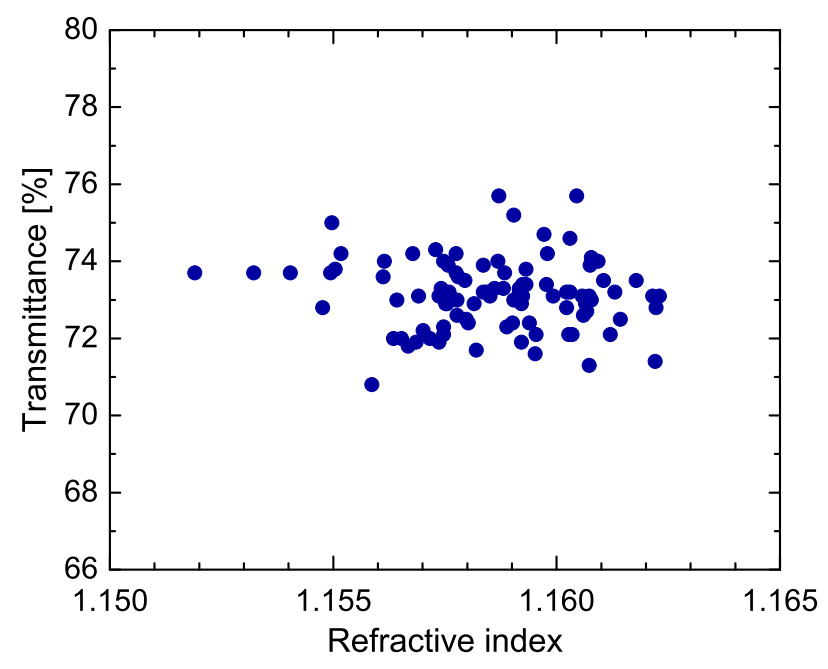

Figure 4: Transmittance at a wavelength of $400 \mathrm{~nm}$ as plotted versus the refractive index. For individual tiles, the refractive indices at the four corners were measured using a 405-nm wavelength laser in air and averaged.

All the manufactured tiles were subjected to a tile-by-tile visual check followed by refractive index and transmittance measurements. The index was determined by measuring the minimum deviation angle of a laser with a wavelength of $405 \mathrm{~nm}$ irradiated to the four corners of the tile. The transmittance along the tile thickness direction was measured over a wavelength range of 200$800 \mathrm{~nm}$ with a spectrophotometer. The detailed setups of these optical measurements have been described in a previous publication [[5], and the initial optical characterization results from the 16 


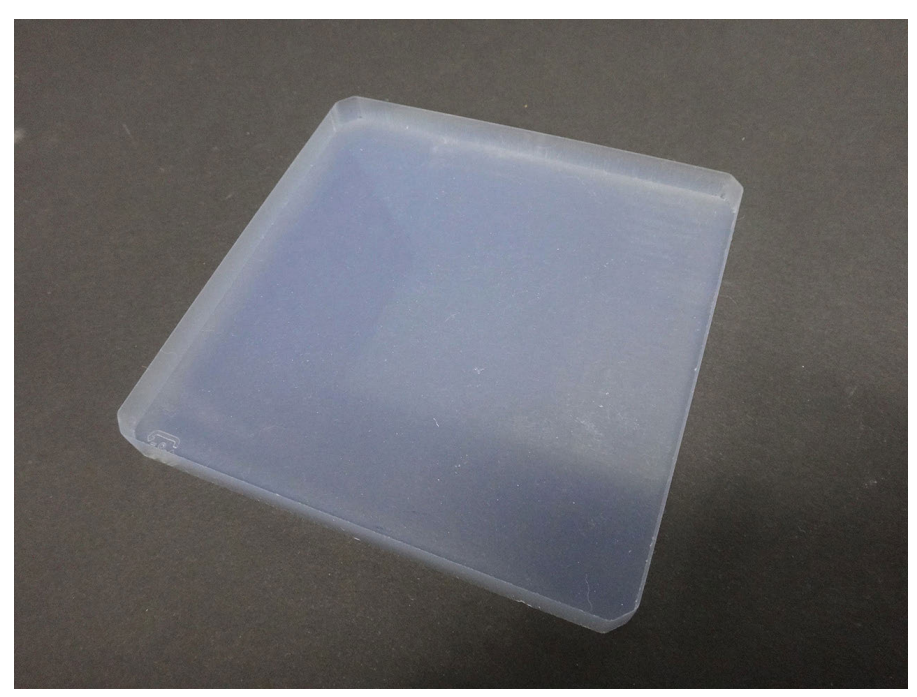

Figure 5: Water-jet-trimmed aerogel tile.

first-batch tiles were also reported previously [6]. Half of the 16 tiles were promising as flight candidate radiators from the viewpoint of the measured transmittance, index, and the integrity of the monolithic tile (i.e., the absence of tile cracking).

Of the 96 tiles, 74 tiles were confirmed to be crack free, resulting in a $77 \%$ yield. The first mass-produced tile with no cracking is shown in Figure $\square$. The tiles typically measured $112 \mathrm{~mm}$ $\times 112 \mathrm{~mm} \times 10 \mathrm{~mm}$. The ultraviolet-visible (UV-vis) transmittance spectrum of the first tile is shown in Figure B. The transmittance at a wavelength of $400 \mathrm{~nm}$ was $74.7 \%$. Figure $\mathbb{\theta}$ shows the transmittance plotted versus the refractive index for the manufactured tiles. One can see that the indices measured for the individual tiles ranged from 1.152 to 1.162 . These measured data will be used for analyzing the physics data. In addition, precisely pre-determining the tile uniformity (i.e., the index and thickness distributions across the tile face) is vital to achieve high-resolution velocity measurements. This task is in progress [ $[\mathbb{d}]$ in parallel to the present study.

\section{Machining and gluing tests}

The aerogel cut processing was validated via a final test performed after the mass production. Each aerogel tile will be installed in a 1-mm-thick aluminum frame with inner dimensions of 100 $\mathrm{mm} \times 100 \mathrm{~mm}$ and attached onto a radiator support plate. Consequently, the tiles will be precisely trimmed using a water-jet cutting device at Tatsumi Kakou Co., Ltd., Japan, making full use of their hydrophobic characteristics. The final machining test was conducted using several tiles from the mass production. By fine-tuning the cutting device operation for the HELIX aerogel tiles, cutting damage at the tile edge was drastically reduced (Figure [5) compared to previous tests [6]. This helps maximize the acceptance area of the radiator. In addition, surface damage caused by aerogel powders generated at the cutting line were minimized by covering the tile with a plastic wrapping film during the machining, allowing the transparency of the tile to remain intact.

The tiles from the machining test were successfully glued into frames (Figure G). The frame was made of anodized aluminum and comprised four tabs at inner edges (see Figure 6 ) to struc- 


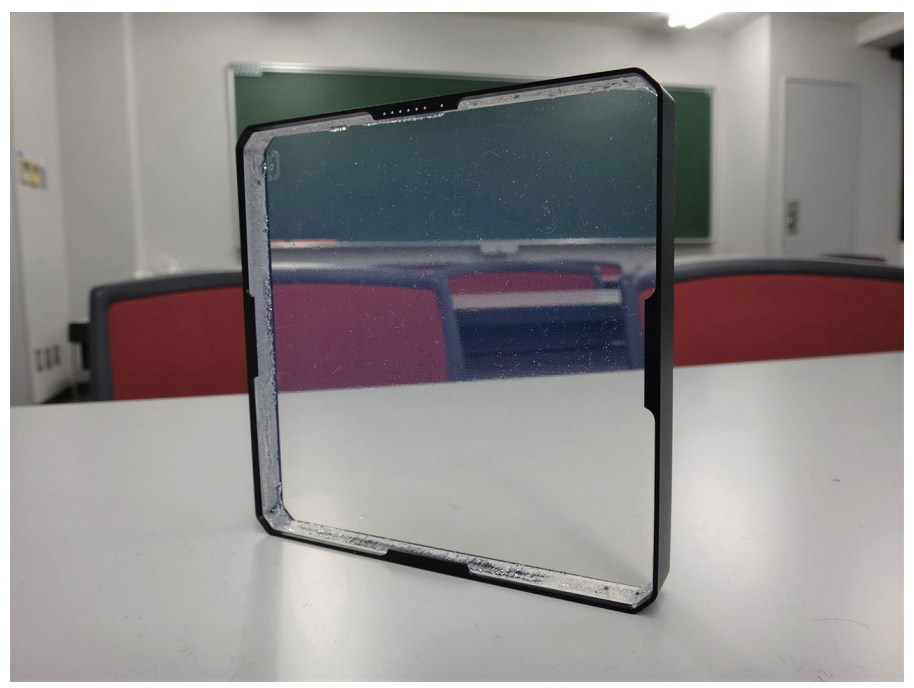

Figure 6: Aerogel tile glued into an aluminum frame.
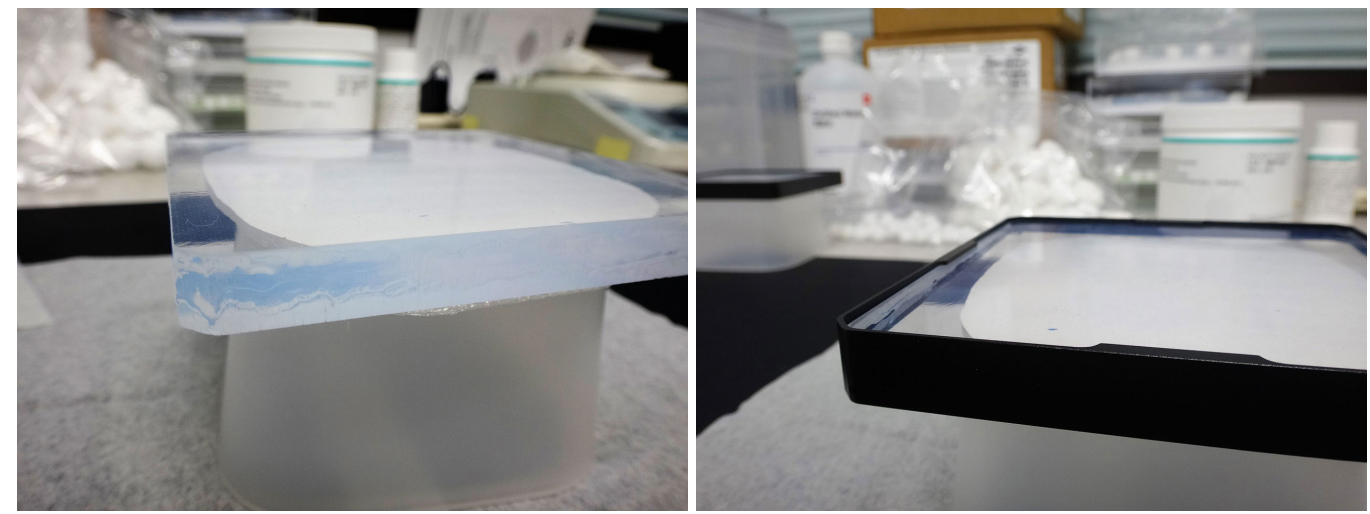

Figure 7: Aerogel-frame gluing procedure. The glue pasted to the edges of the tile (left) and the frame fitted along the edges of the tile (right).

turally support the tiles from the downstream (i.e., photosensor) side. A silicone elastomer (Sylgard 184, Dow Silicones Corporation, USA), which was validated during a previous balloon mission [8], was chosen as a glue to fix the trimmed tiles into the frames. As shown in Figure $\mathbb{Z}$, the glue was pasted to the edges of the tile using a plastic spatula, and the frame was fitted along the edges of the tile. The frame was supported and fixed on the tile using the tabs in this step, which was maintained for $48 \mathrm{~h}$ to sufficiently cure the glue. In an overload test on the tile surface, non-negligible damage by the glue was observed; however, this damage could be sufficiently minimized by reducing the amount of glue used. Thus, a procedure for securely gluing the tiles into the frames was established.

\section{Conclusions}

The mass production of 96 hydrophobic silica aerogel tiles with a refractive index of $\sim 1.15$ was completed, yielding 74 crack-free tiles. In the initial optical assessment, sufficient transmit- 
tance (greater than $70 \%$ at a wavelength of $400 \mathrm{~nm}$ ) was confirmed. The water-jet cutting and gluing tests were successful; therefore, the processing method for the flight aerogel tiles is ready. After investigations concerning the uniformity of the tiles, the aerogel radiators will be installed in the HELIX RICH detector.

\section{Acknowledgments}

This study was supported by a Grant-in-Aid for Scientific Research (C) (No. 18K03666) from the Japan Society for the Promotion of Science (JSPS).

\section{References}

[1] S. P. Wakely et al., Prospects for high energy light isotope measurements on balloons, in proceedings of the 34th International Cosmic Ray Conference, POS(ICRC2015) 682.

[2] P. Allison et al., Cosmic-ray isotope measurements with HELIX, in proceedings of the 36th International Cosmic Ray Conference, POS (ICRC2019) 121.

[3] P. Allison et al., Design and construction of the HELIX RICH detector, in proceedings of the 36th International Cosmic Ray Conference, POS (ICRC2019) 152).

[4] M. Tabata et al., Development of transparent silica aerogel over a wide range of densities, Nucl. Instrum. Methods Phys. Res. A 623 (2010) 339-341.

[5] M. Tabata et al., Hydrophobic silica aerogel production at KEK, Nucl. Instrum. Methods Phys. Res. A 668 (2012) 64-70.

[6] M. Tabata et al., Developing a silica aerogel radiator for the HELIX ring-imaging Cherenkov system, Nucl. Instrum. Methods Phys. Res. A (2019), in press. https://doi.org/10.1016/j.nima.2019.02.006

[7] P. Allison et al., Calibration of the aerogel tiles for the HELIX RICH, in proceedings of the 36th International Cosmic Ray Conference, POS (ICRC2019) 133.

[8] A. W. Labrador et al., Silica aerogel Cherenkov counters for the Isotope Matter-Antimatter Experiment, in proceedings of the 23rd International Cosmic Ray Conference, vol. 2 (1993) pp. 524-527. 